\title{
L'Afrique, le nouveau continent milliardaire
}

2. LES DENSITÉS LES PLUS

ÉLEVÉES ET LES PLUS FAIBLES EN AFRIQUE

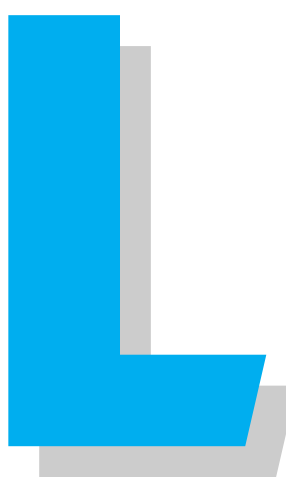

\section{par Gérard-François DUMONT}

a répartition traditionnelle $\mathrm{du}$ monde en cinq continents conduit à opposer l'Asie, où habite $60 \%$ de la population du monde ${ }^{1}$, et le très faible peuplement de l'Océanie $^{2}$. Entre ces extrêmes, le rang respectif de l'Europe, de l'Amérique et de l'Afrique évolue. Selon les estimations, l'Afrique vient de dépasser le seuil du milliard d'habitants à l'orée des années 2010.

Pendant longtemps, encore au XIXe siècle et pendant la majeure partie du XX' $X^{\mathrm{e}}$, la population de l'Europe ${ }^{3}$ est supérieure à celle de l'Amérique et davantage encore à celle de l'Afrique. Compte tenu des effets de la transition démographique 4 en Amérique latine, de l'attractivité migratoire de l'Amérique du Nord et d'une fécondité qui remonte aux États-Unis, la population de l'Amérique devient plus nombreuse que celle de l'Europe dès 1991.

Six plus tard, en 1997, l'Europe est dépassée par l'Afrique. Malgré l'immigration en Europe, la fécondité très affaiblie d'une Europe en " hiver démographique » conduit à une stagnation démographique. En revanche, en Afrique, malgré l'importance de l'émigration, la croissance démographique est portée par la logique de la transition démographique.

Puis, en 2004, le rythme démographique de l'Afrique conduit ce continent à un peuplement supérieur à celui de l'Amérique pour franchir le cap du milliard d'habitants en 2010. Néanmoins, cette information doit être analysée de façon plus fine.

\section{LA POPULATION DE L'AFRIQUE} COMPARÉE À CELLE DE L’AMÉRIQUE ET DE L'EUROPE (RUSSIE COMPRISE)

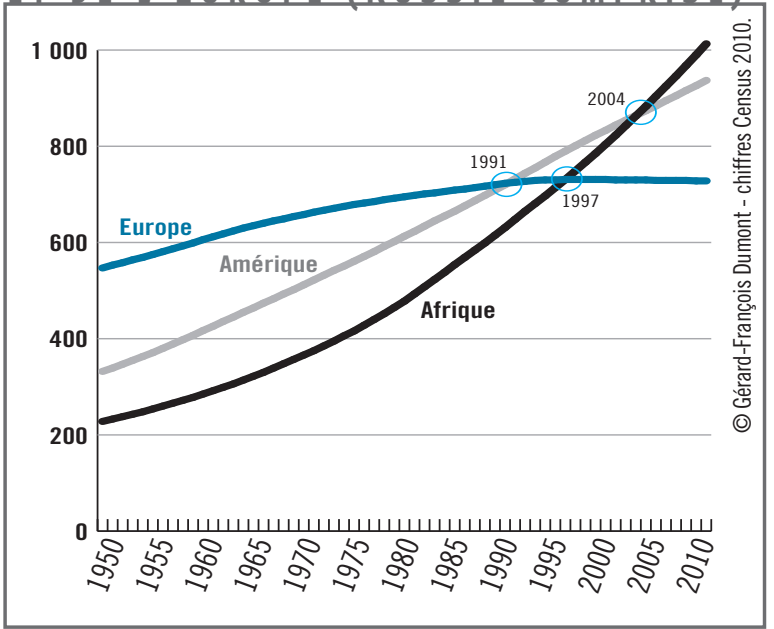

Le milliard d'habitants annoncé en Afrique ne peut être considéré que comme un ordre de grandeur reposant sur des estimations susceptibles d'être révisées. En effet, nombre de pays d'Afrique subsaharienne n'ont pas organisé de recensement de la population depuis plusieurs décennies et leur nombre d'habitants annoncé repose donc sur des évaluations. Ces dernières sont rendues difficiles dans la mesure où l'état civil est insuffisant. Par exemple, en Afrique subsaharienne, près de deux enfants sur trois n'étaient pas enregistrés en 2007. Dans six pays de cette région, le pourcentage d'enfants de moins de 5 ans enregistrés était inférieur ou égal à $10 \%{ }^{5}$.

En second lieu, l'arrivée du nombre d'habitants de l'Afrique à un chiffre dépassant le milliard pourrait donner l'impression d'un continent très peuplé. Or, en réalité, la densité de la population de l'Afrique est, en 2010, de 34 habitants $/ \mathrm{km}^{2}$, inférieur à la moyenne mondiale de 51 . En réalité, les niveaux de densité des 60 pays d'Afrique sont fort différents. S’opposent par exemple les densités élevées de territoires îliens (Maurice, Mayotte et les Comores), du Rwanda et du Burundi, aux densités extrêmement faibles du Gabon, de la Libye, du Botswana, de la Mauritanie et de la Namibie.

En outre, le potentiel démographique des pays d'Afrique est fort différencié selon le rythme de leur mouvement naturel ou de leur mouvement migratoire. La croissance possible des pays d'ici 2025 varie par exemple de près de $80 \%$ au Niger contre moins de $8 \%$ en Afrique du Sud ${ }^{6}$.

Ainsi, le chiffre d'un milliard, s'il témoigne des effets de la transition démographique en Afrique depuis la seconde moitié $d u X X^{e}$ siècle, ne doit pas masquer le caractère très diversifié de la géographie de la population africaine ${ }^{7}$.

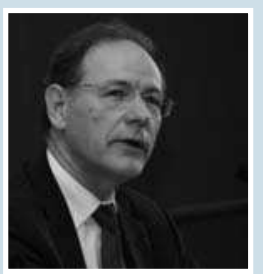

1. 4117 millions d'habitants en 2009.

2. 36 millions d'habitants en 2009.

3. Russie comprise

4. « Période, de durée et d'intensité variables, pendant laquelle une population passe d'un régime démosshique de morta-

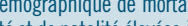
tee et de natalité élevées à un régime de basse mortalité, puis de faible natalité ». Cf. Dumont, GérardFrancoois, Les populations du monde, Paris, Éditions Armand Colin, deuxième édition, 2004.

5. Progrès pour les enfants, Unicef, $n^{\circ} 8$, sepentants, Unicef
tembre 2009 .

6. « La population des continents et des États en 2009 ", Population \& Avenir, no 695 , novembredécembre 2009

7. Cf. Wackermann, Gabriel (direction), L'Afrique en dissertations corrigées, Paris, Ellipses, 2004 . Frérot, Anne-Marie (direction), L'Afrique en questions, Paris, Ellipses, 2004.

$$
\begin{aligned}
& \text { Toujowis soncieuse dêtre à la pointe dans une analyse claire } \\
& \text { et argumentée des populations et des territoires, } \\
& \text { la revue QPopulation of Chenir vous remercie de votre fiététité } \\
& \text { et rous souhaite une bonne année } 2010 .
\end{aligned}
$$

GexO 